\title{
BIM: Os desafios tecnológicos e inovação profissional da engenharia civil no estado
}

\section{do Maranhão}

BIM: The technological challenges and professional innovation of civil engineering in the state of

\section{Maranhão}

BIM: Los retos tecnológicos y la innovación profesional de la ingeniería civil en el estado de

\section{Maranhão}

Recebido: 08/12/2021 | Revisado: 13/12/2021 | Aceito: 20/12/2021 | Publicado: 24/12/2021

\author{
Witson Andrade da Silva \\ ORCID: https://orcid.org/0000-0001-5155-7579 \\ Universidade Federal do Maranhão, Brasil \\ E-mail: eng.silva10@hotmail.com \\ Gleidson Ferreira Fonseca \\ ORCID: https://orcid.org/0000-0002-6543-6310 \\ Universidade Federal do Maranhão, Brasil \\ E-mail: gleidson.fonseca@discente.ufma.br \\ Wener Ferreira Silveira \\ ORCID: https://orcid.org/0000-0003-4032-7369 \\ Universidade Federal do Maranhão, Brasil \\ E-mail: wener.silveira@discente.ufma.br
}

\begin{abstract}
Resumo
O foco principal deste artigo é o desafio tecnológico como um elemento aplicado na indústria da construção e especialmente na engenharia civil. Com este cenário em mente, o artigo analisa os benefícios, contribuições e desafios da implementação desta tecnologia avançada e como ela pode e irá melhorar os padrões organizacionais das empresas, além de ajudar a melhorar o desempenho da empresa e a qualidade do produto oferecido aos empreiteiros. Com a evolução das ferramentas tecnológicas, hoje podemos dizer que a implementação do BIM (Building Information Modeling) está presente, o que significa uma evolução dos processos convencionais (CAD), nas práticas de projeto, produção, gerenciamento e entrega de projetos e obras arquitetônicas, mas também uma revolução, tanto no mercado quanto na educação e nas práticas de engenharia e arquitetura. É de suma importância treinar profissionais com habilidades e habilidades para trabalhar com esta nova plataforma e depois ser capaz de liderar este processo de mudança na indústria da construção.
\end{abstract}

Palavras-chave: Tecnologia; Engenharia civil; Inovações; Projetos.

\begin{abstract}
The main focus of this article is the technological challenge as an applied element in the construction industry and especially in civil engineering. With this scenario in mind, the article analyzes the benefits, contributions and challenges of implementing this advanced technology and how it can and will improve the organizational standards of companies, as well as help improve company performance and the quality of the product offered to contractors. With the evolution of technological tools, today we can say that the implementation of BIM (Building Information Modeling) is present, which means an evolution of conventional processes (CAD), in the practices of design, production, management and delivery of architectural projects and works, but also a revolution, both in the market and in the education and practices of engineering and architecture. It is of paramount importance to train professionals with skills and abilities to work with this new platform and then be able to lead this process of change in the construction industry.
\end{abstract}

Keywords: Technology; Civil engineering; Innovations; Projects.

\section{Resumen}

El enfoque principal de este artículo es el reto tecnológico como elemento aplicado en la industria de la construcción y especialmente en la ingeniería civil. Con este escenario en mente, el artículo analiza los beneficios, las aportaciones y los retos de la implantación de esta tecnología avanzada y cómo puede y va a mejorar los estándares organizativos de las empresas, además de ayudar a mejorar el rendimiento de las mismas y la calidad del producto ofrecido a los contratistas. Con la evolución de las herramientas tecnológicas, hoy podemos decir que está presente la implantación del BIM (Building Information Modeling), que supone una evolución de los procesos convencionales (CAD), en las 
prácticas de diseño, producción, gestión y entrega de proyectos y obras de arquitectura, pero también una revolución, tanto en el mercado como en la educación y las prácticas de ingeniería y arquitectura. Es de suma importancia formar profesionales con habilidades y destrezas para trabajar con esta nueva plataforma y así poder liderar este proceso de cambio en la industria de la construcción.

Palabras clave: Tecnología; Ingeniería civil; Innovaciones; Proyectos.

\section{Introdução}

A construção civil desempenha um papel fundamental na economia mundial, mas há muito que se caracteriza por uma baixa produtividade. Os seus processos são largamente fabricados, dando origem a baixa eficiência, elevado consumo e desperdício de recursos materiais e humanos.

Com a utilização de programas que auxiliariam os profissionais no desenho técnico auxiliado por computador CAD 2D (Computer Aided Design) no início da década de 1980, a construção civil ganhou um novo status quanto a sua produtividade no desenvolvimento de projetos, mas, apesar disso, essa tecnologia ainda continua sendo complexa para aqueles que não estão acostumados a trabalhar com a ferramenta.

Há pouco tempo, está sendo utilizado o Building Information Modeling - Modelagem de Informações da Construção (BIM), que gera uma enorme quantidade de especificações ao projeto, uma vez que é compatibilizado várias informações ao mesmo tempo ao trabalho realizado, que por sua vez é compartilhado por todos os profissionais envolvidos.

Neste contexto, o BIM surge como um método que pode trazer várias melhorias no sector da construção como um todo e reduzir ou mesmo eliminar várias causas de baixa produtividade.

O Empenho no BIM, um acrónimo de (Building Information Modeling), uma tecnologia que permite a criação de modelos virtuais 3D e organiza a informação sobre obras e construção civil de forma automatizada e precisa, permitindo um controlo e gestão mais eficientes do que as plataformas utilizadas até agora:

"BIM é uma filosofia de trabalho que integra arquitetos, engenheiros e construtores (AEC) na elaboração de um modelo virtual preciso, que gera uma base de dados que contém tanto informações topológicas como os subsídios necessários para orçamento, cálculo energético e previsão de insumos e ações em todas as fases da construção" (Eastman, 2008).

Com a adoção desse novo método, existem ainda alguns problemas e desafios a ultrapassar na utilização eficaz do BIM, tais como a dificuldade das partes envolvidas em aceitar as mudanças inerentes aos processos de trabalho do BIM, que até então eram dominados por determinadas, e a comunicação ineficiente que impede o trabalho em colaboração, que é um dos pilares do BIM. Além disso, existe uma falta de conhecimento sobre o próprio processo: a utilização de novas tecnologias, como colaborar com outros profissionais, fraca visão multidisciplinar, falta de conhecimento sobre modelização e falta de interoperabilidade.

O conceito BIM está centrado na informação ou na modelação da informação. Por conseguinte, todos os autores envolvidos na concepção de um projeto de construção, seja ele novo ou renovado, devem ser incorporados no conceito BIM. BIM inclui não só os designers profissionais envolvidos na fase de concepção de um projeto, mas também os envolvidos no processo de planeamento, execução e gestão.

\section{Revisão da Literatura}

\subsection{Planos de implementação BIM}

A função principal deste documento é de fornecer uma visão geral sobre metas e diretrizes para implementação das melhores práticas do Building Information Modeling (BIM).

BIM ajuda as partes interessadas a tomar decisões informadas, melhorando a comunicação e colaboração entre as 
equipas. De acordo com um relatório da CBIC, $87 \%$ dos utilizadores do BIM revelaram que receberam benefícios positivos da implementação do BIM e o BIM ajudou-os a entrar:

- Redução de erros

- Prever com precisão o custo do projeto

- Melhorar a sua compreensão do projeto

- Definição de linhas de tempo transparentes

- Melhorar os esforços de programação do projeto

O fluxo de trabalho baseado no BIM pode ser extremamente benéfico para as empresas modernas de AEC, mas para implementar eficazmente o BIM, toda a organização precisa de adoptar uma abordagem estruturada. O primeiro passo para começar com o BIM é lidar com o preconceito e o medo de transição de uma abordagem convencional.

O Governo Federal brasileiro criou iniciativas para a divulgação e democratização do BIM, tanto através de decretos como através da Estratégia BIM BR, cujos objetivos também incluem a estruturação do setor público para a adoção do BIM; a capacitação dos profissionais envolvidos; o desenvolvimento e aplicação de tecnologias relacionadas ao BIM; a criação de atos normativos que estabelecem parâmetros para a contratação e contratação de obras públicas no BIM, bem como o desenvolvimento de normas técnicas, guias e protocolos específicos para a implementação da metodologia.

Foi lançado em 16/05/2018, uma estratégia para promover um aperfeiçoamento na indústria da construção civil. A Estratégia BIM BR, instituída pelo Decreto nº 9.377, de 17 de maio de 2018.

Art. $4^{\circ} \mathrm{A}$ implementação do BIM ocorrerá de forma gradual, obedecidas às seguintes fases:

I - Primeira fase - a partir de $1^{\circ}$ de janeiro de 2021, o BIM deverá ser utilizado no desenvolvimento de projetos de arquitetura e engenharia, referentes a construções novas, ampliações ou reabilitações, quando consideradas de grande relevância para a disseminação do BIM, nos termos do disposto no art. 10, e abrangerá, no mínimo:

a) a elaboração dos modelos de arquitetura e dos modelos de engenharia referentes às disciplinas de:

1. estruturas;

2. instalações hidráulicas;

3. instalações de aquecimento, ventilação e ar condicionado; e

4. instalações elétricas;

b) a detecção de interferências físicas e funcionais entre as diversas disciplinas e a revisão dos modelos de arquitetura e engenharia, de modo a compatibilizá-los entre si;

c) a extração de quantitativos; e

d) a geração de documentação gráfica, extraída dos modelos a que se refere este inciso;

II - Segunda fase - a partir de $1^{\circ}$ de janeiro de 2024, o BIM deverá ser utilizado na execução direta ou indireta de projetos de arquitetura e engenharia e na gestão de obras, referentes a construções novas, reformas, ampliações ou reabilitações, quando consideradas de grande relevância para a disseminação do BIM, nos termos do disposto no art. 10, e abrangerá, no mínimo:

III - Terceira fase: a partir de $1^{\circ}$ de janeiro de 2028, o BIM deverá ser utilizado no desenvolvimento de projetos de arquitetura e engenharia e na gestão de obras referentes a construções novas, reformas, ampliações e reabilitações, quando consideradas de média ou grande relevância para a disseminação do BIM, nos termos do disposto no art. 10, e abrangerá, no mínimo:

- Documentação do plano BIM e avaliação do projeto piloto:

Enquanto se trabalha no projeto piloto, é essencial documentar tudo, desde o processo BIM até às diferenças de 
produtividade devido à transição. Nesta fase, é também importante compreender as expectativas dos outros intervenientes na cadeia de valor.

Em vez de avançar diretamente com níveis definidos de BIM ou padrões novos, é importante compreender o seu papel no ciclo de vida do BIM. Uma vez que o BIM tem tudo a ver com colaboração e comunicação, trazer todas as partes interessadas desde o início ajudará a longo prazo.

Além disso, é importante não só medir a sua produtividade, realização de objetivos e ajustar o seu roteiro, mas também documentar os obstáculos enfrentados durante a adoção do BIM no projeto piloto. Isto permitirá à sua equipa e gestão encontrar soluções valiosas para as lutas enfrentadas durante a adoção do BIM.

\subsection{Soluções para coordenação, implementação e gestão}

Um estudo do Instituto de Engenheiros Civis do Reino Unido e o Grupo de Tarefa BIM do governo do Reino Unido revelou dados importantes do uso do BIM na construção civil: mais de $80 \%$ dos profissionais que passaram a adotar a metodologia tiveram uma percepção de mais eficiência e economia em seus projetos.

Uma importante ressalva é que o Reino Unido é um dos países mais avançados no uso do BIM na construção civil pelo mundo, trazendo inúmeras experiências bem-sucedidas e estatísticas importantes para o setor.

O BIM surge como uma verdadeira inovação dentro da cadeia construtiva, que traz diversos benefícios. (Vitor, 2021)

O BIM aparece, hoje, como uma necessidade e uma obrigação, levando as pessoas e organizações a se adaptarem cada vez mais rapidamente a esta nova realidade e à mudança que ela implica. No entanto Gonçalves Jr. Fala que:

Projetos em BIM exigem mudanças de paradigmas no que diz respeito as pessoas, processos e tecnologia, uma mudança no workflow, na qual saímos de um modelo 2D para um modelo tridimensional repleto de informações totalmente integradas. A mudança nos remete a diversos desafios, na qual apenas a entrega de um modelo BIM não garante o pleno uso e benefícios que a metodologia propõe e um sucesso na sua implantação.

Essa implementação feita a esmo pode gerar uma frustração, pois uma mudança sem planejamento, pode gerar resultados desagradáveis, atrasos nas entregas, perda de produtividade, desmotivação da equipe. (Gonçalves Jr., 2019)

De uma situação de aplicações pontuais e erráticas, passamos a uma generalização do conceito e de sua globalização. Hoje, uma grande porcentagem dos atores da indústria de Arquitetura, Engenharia, Construção e Operação (AECO) já sabe como identificar seus princípios básicos, aplicações e usos. Com a imensidão de inovações tecnológicas somos surpreendidos muitas vezes por um grande número de casos de aplicação local, eventual, disseminando a metodologia do BIM, entre outros, veiculada pela comunicação via web, tem sido muito eficaz.

Em todo caso, para muitos, porém, surge a necessidade de decodificar um novo léxico, de aprender novas ferramentas e de implementar novos procedimentos.

\subsubsection{BIMMS - Management Solutions}

Em 2014 foi criada A BIMMS - Management Solutions, com a missão de incentivar os parceiros industriais da AECO a integrar e utilizar a metodologia BIM em seus processos de criação, atribuindo suas habilidades técnicas e profissionais, a serviço de projetos globais de arquitetura, engenharia e construção.

A consultoria BIM é ainda uma atividade muito recente que precisa ser mais estudada do ponto de vista científico, por isso ainda é difícil encontrar estudos específicos sobre este assunto. Ela se baseia essencialmente no conhecimento e experiência dos consultores que, diante de uma nova abordagem dos métodos tradicionais de projeto, construção e operação, escolhem as soluções e implementações que parecem mais eficientes e apropriadas. Embora muita teoria possa ser encontrada sobre o BIM e seus usos e aplicações, sua aplicação prática não é tão fácil de encontrar. Para isso, coletamos alguns estudos dos consultores do BIMMS, os usos e aplicações adotadas em vários casos práticos internacionais. A dificuldade em superar as limitações de confidencialidade inerentes aos projetos, muitos deles ainda em andamento, impediu seu referenciamento 
nominal.

A partir dos estudos realizados, percebe-se uma lógica holística, que percorre todo o processo desde a modelagem, coordenação, implementação, treinamento e até mesmo inovação.

\subsubsection{AECO - (Arquitetura, engenharia, construção e operação)}

Atualmente, a indústria AECO enfrenta novos desafios de grande relevância, tais como a crescente complexidade dos projetos a serem executados, a necessidade de praticar opções sustentáveis, preocupações energéticas globais, redução de custos e melhoria da produtividade, entre outros. Como resposta à necessidade de melhorar os atuais processos de trabalho dos projetos, surgiu o conceito BIM, que se baseia essencialmente em uma metodologia de construção virtual que, entre muitas outras capacidades, permite o compartilhamento de informações entre todos os atores e fases do projeto. Em um projeto utilizando a metodologia BIM, a compatibilização é feita por relações paramétricas, o que resulta em mudanças realizadas em tempo real por todo o modelo, dificultando o desenvolvimento é de erros e agilizando os processos de atualização.

A fim de implementar plenamente o processo BIM, baseado em mapas de processo claramente identificados, detalhados e abrangentes que definem o fluxo de informações, a tecnologia permite várias melhorias em cada fase do ciclo de vida de um projeto. O BIM fornece visualizações mais antigas, colaboração eficaz de múltiplas disciplinas de projeto, extração de estimativas de custo, deteç̧ão de erros e omissões antes da construção, que melhoram a pré-construção e o projeto.

O BIM tem sido avaliado como benéfico para a construção, fabricação e pós-construção, principalmente devido ao uso do modelo de projeto como base para a fabricação de componentes, sincronização de projeto e planejamento da construção e integração com Sistemas de Gerenciamento de Operações e Instalações. A implementação prática desta metodologia está começando a ser evidente, mas ainda tem que lidar com dificuldades, ou seja, aquelas inerentes à curva de investimento e aprendizado. A necessidade de adquirir habilidades a nível profissional começa a ser sentida, tanto em arquitetura, engenharia civil e cursos especiais, como também no ambiente empresarial.

Esta transição para o BIM trouxe várias mudanças na maneira de fazer e pensar em diversas disciplinas. A arquitetura tem à sua disposição auxílios para o projeto, produção e comunicação de sua proposta que antes não existiam. A engenharia começa a se integrar com os processos de fabricação e automação para otimizar suas soluções. A construção se adapta a novos modelos empresariais e de contratação, ao trabalho colaborativo e à integração de vários sistemas de gestão. Operação e manutenção integram o conhecimento das fases anteriores, visando um ciclo de vida mais sustentável.

A partir da coleta de vários casos práticos nos quais o BIMMS participou desde seu início, foram identificados os principais usos que integram este ciclo de vida, optando por agrupá-los em Modelagem, Coordenação, Implementação, Treinamento e Inovação, conforme apresentado nos capítulos seguintes.

\subsubsection{Modelagem e coordenação}

A modelagem é o elemento básico a partir do qual a interação e a integração de informações podem começar. Este processo de Modelagem, como a Construção, foi desenvolvido como um agrupamento de Peças, Materiais e Equipamentos, que concentra a atenção do Designer na solução e não no desenho ou na representação.

Nos trabalhos analisados, a preocupação é que os elementos modelados tenham propriedades geométricas e nãogeométricas, estas propriedades juntas constituem um banco de dados automático de informações inerentes ao projeto que pode ser utilizado por qualquer projetista durante o ciclo de vida do edifício em qualquer etapa do Projeto à Operação e Manutenção. Os principais modelos necessários são os modelos arquitetônicos, estruturais, hidráulicos (abastecimento, drenagem, água da chuva, rede de incêndio), mecânicos e elétricos. Se já existem algumas bibliotecas que disponibilizam estes componentes, especialmente quando se trata das grandes marcas, ainda há muito a ser desenvolvido e é necessário programar estes blocos de 
classes e famílias.

Igualmente recorrente é a solicitação de modelos que são desenvolvidos a partir do levantamento existente, seja a partir de desenhos, levantamentos topográficos ou aquisição de nuvens de pontos. Nesses modelos de registro, normalmente é admitido um nível inferior de informação, correspondente à falta de conhecimento das características dos materiais e processos de construção inicialmente utilizados.

Inerente à produção do modelo está sua utilização para a simulação de formulários, processos e análises, assim como a respectiva comunicação.

Os desafios apresentados à Arquitetura e Engenharia com a concepção e adoção de formas e ideias menos convencionais, tornam-se mais fáceis de serem superados através de sua visualização. Desde a fase de concepção até a execução, o alinhamento entre a equipe do projeto é fortalecido com a visualização tridimensional. O desenvolvimento deste processo é evolutivo, agregando valor a cada fase do projeto através da simulação da realidade.

A produção de renders e os trabalhos de pós-produção arquitetônica visam contribuir tanto para a comunicação das ideias quanto para a simulação do ambiente a ser construído. No processo de criação do projeto, neste processo de simulação, um dos usos mais consideráveis e mais fundamentais é a retirada de peças desenhadas diretamente do modelo. A necessidade de criar desenhos com uma linguagem visual compreensível e compatível com a padronização da empresa requer um esforço de configuração e ajuste nos diversos softwares e plataformas que não deve ser negligenciado.

Da mesma forma, a extração de quantidades requer uma verificação cuidadosa da qualidade do modelo, de modo a não incorrer em erros. Em fases conceituais, esta extração pode ser muito útil, mas às vezes não dispõe de informações suficientes para atingir o objetivo pretendido.

O conceito BIM realmente começa a fazer sentido quando o componente de colaboração entre os vários atores é incluído, e, portanto, entre os vários modelos, implicando sua cuidadosa coordenação.

Esta coordenação tem diferentes níveis e graus de intervenção, porém, em casos mais avançados, ela começa com a gestão da informação. O domínio de dados, com a organização da criação, análise e controle de documentos, é essencial para que a estrutura de informação seja gerenciável.

Aparentemente sendo algo simples, sua aplicação prática, dados os milhares de conflitos que às vezes existem, assim como a necessidade de encontrar soluções concretas para cada um destes problemas, faz desta uma atividade altamente especializada que requer experiência. Outro aspecto muito comum em quase todos os casos analisados, onde a coordenação se torna mais eficaz, é a adoção de um ambiente colaborativo comum. A possibilidade de utilizar plataformas de compartilhamento de arquivos na nuvem ou servidores locais em tempo real torna possível que durante a modelagem, todas as especialidades sejam visíveis, detectando antecipadamente possíveis problemas de organização, espaço ou conflito.

Associados a esta questão de coordenação estão, muitas vezes, problemas de interoperabilidade. A existência de diferentes plataformas e softwares, assim como a troca de informações em diferentes formatos, leva à necessidade de controlar estes problemas, sempre com o objetivo de obter um modelo federado coordenado. Para a verificação final e validação da qualidade deste modelo, pode ser utilizado software que, quando programado, pode suportar a verificação da solução recomendada para conflitos ou para verificar a solução sob condições regulatórias, conforto ou mesmo construtibilidade. Nessas trocas de informações e protocolos, a necessidade de incorporar formatos de gestão de operações e manutenção, ou seja, COBIE.

\subsubsection{Implementação e gestão}

O processo de implementação e treinamento do BIM é geralmente muito mais caro do que a aquisição da Tecnologia. Os processos tradicionais têm sido os mesmos por muitos anos, estão enraizados e muitas vezes otimizados ao máximo, mas 
limitados para os dias de hoje.

Após a análise de estudos, a etapa de mudança é frequentemente dificultada por anos e anos de conhecimento adquirido em processos mecanizados pelos usuários, e esta é a barreira mais difícil de ser superada. O apoio de uma empresa especializada em implementação torna-se fundamental não apenas para que a curva de motivação/aprendizagem da equipe não caia para níveis que comprometam o sucesso da implementação, mas também para que eles se beneficiem do apoio de um conjunto necessário de conhecimentos técnicos e experiência de uma equipe especializada que os oriente da melhor maneira, para que os processos sejam rapidamente estabilizados.

Dentro do BIMMS, as habilidades e perfis dos funcionários designados para estas áreas de implementação e treinamento diferem substancialmente daqueles necessários para a modelagem e coordenação. A exigência de contato pessoal com o cliente e a vontade de aprender a cultura das organizações exige perfis de consultores com sólidos conhecimentos técnicos, mas com experiência bem desenvolvida e soft-skills de relacionamento. O uso da metodologia BIM foi iniciado na fase de projeto, permitindo assim que durante a fase de construção haja uma transmissão do conhecimento adquirido nas fases passadas e uma evolução no Banco de Dados contido no Modelo BIM.

Em algumas aplicações mais avançadas, o suporte de contratação BIM, bem como a consequente verificação do cumprimento das Normas BIM e do Planejamento de Execução BIM, assume importância crucial. Sem estas ferramentas de controle e supervisão, o processo não é desenvolvido.

Uma das fundamentais serventias que o BIMMS tem a prestar com seus parceiros, tem sido um suporte técnico in situ, seja no local ou em um escritório. Começando com a preparação do trabalho e verificação/adaptação do projeto às restrições do contrato, métodos de trabalho e planejamento. No decorrer deste tempo, o modelo BIM serve como base fundamental de trabalho para a construção, permitindo a extração de desenhos, além do planejamento de elementos que deverão ser préfabricados, bem como a preparação da cofragem e simulação da construção. Todos os intervenientes trabalham sob a mesma referência, permitindo uma fácil compreensão da solução recomendada e das possíveis alternativas.

A Construção Virtual permite novos processos de trabalho como, por exemplo, a preparação dos trabalhos em visualizações 3D, a validação dos relatórios de medição pelo modelo ou mesmo as reuniões de trabalho/trabalho em que o Modelo é visualizado, evitando assim deslocamentos e movimentos de pessoas para o local. O georreferenciamento de todos os elementos permite um monitoramento constante do processo de construção. Ferramentas de escaneamento a laser podem ser usadas para acompanhar a fase de construção ou mesmo para validar a construção no nível geométrico.

Os pedidos de esclarecimento suportados pelos modelos e plataformas BIM, são um dos usos mais necessários quando o BIMMS fornece suporte técnico no local. A facilidade com que estas consultas são complementadas com ilustrações extraídas do modelo, traz uma nova abordagem à forma como os inspetores foram usados para responder a tais solicitações dos construtores.

Igualmente importantes, mas mais difíceis de obter, são os desenhos de preparação técnica, extraídos diretamente do modelo. A dificuldade em detalhar o modelo tem uma correspondência direta com o grau de detalhe que pode ser obtido a partir destes desenhos.

A gestão do trabalho implica uma grande conivência com o Gerente BIM, a fim de controlar efetivamente tanto o tempo quanto os custos inerentes a ele. No processo de planejamento, o modelo BIM 4D é utilizado para representar graficamente tanto elementos permanentes como temporários com o tempo das atividades de construção. Ele pode incluir recursos de mão-de-obra, materiais e entregas associadas, e a localização dos equipamentos.

Como os componentes do modelo 3D estão diretamente ligados à programação, a visualização do planejamento e dos recursos pode ser analisada em diferentes espaços temporais e físicos. O modelo 4D BIM também pode ser usado para planejar efetivamente as fases de construção, controlar o trabalho realizado diariamente e monitorá-lo em relação ao planejamento 
inicial, bem como gerenciar espaços ocupados, caminhos de acesso, e também apoiar a implementação de planos de segurança e monitoramento e prevenção ambiental no local. O uso de ferramentas de gerenciamento e planejamento apoiadas pelos modelos BIM está se tornando um procedimento essencial nas empresas de construção, pois seu uso promove uma redução do risco nas obras contratuais, bem como uma maneira fácil de criar cenários de simulação do planejamento da construção.

Análise de custos (BIM 5D) ou controle orçamentário pode ser usado desde a fase inicial até a conclusão do trabalho, servindo como elemento de gestão apoiado por quantidades retiradas diretamente do modelo. Ao associar custos ao planejamento, é possível extrair facilmente cronogramas financeiros, gráficos de fluxo de caixa, relatórios de medição e outros, que são fundamentais para qualquer empresa de construção.

Inerente a estes processos de implementação, foram desenvolvidas ações de treinamento com o objetivo de melhorar as habilidades de acordo com os papéis e perfis de cada pessoa envolvida. É percebido a partir dos diversos processos, que estamos caminhando para sistemas de certificação para profissionais e empresas, que serão fatores condicionantes para a participação em licitações e obras.

Dos vários tipos de treinamento que o BIMMS foi solicitado a coordenar, o treinamento genérico para o alinhamento e envolvimento do pessoal na causa BIM, surge como o fator inicial e geralmente condicionante para todo o acompanhamento. Isto é seguido por treinamentos gerais e departamentais e sessões de alinhamento organizacional sobre o BIM. Muitas vezes são necessárias sessões técnicas para adquirir habilidades em software e ferramentas BIM básicas e avançadas. Treinamentos práticos de verificação são geralmente no local e servem como controle e acompanhamento dos processos BIM.

\subsubsection{Inovação}

Por último, mas com forte repercussão na sustentabilidade, é a inovação, na qual qualquer consultor deve investir pesadamente. A passagem de seus conhecimentos para seus parceiros deve resultar no reconhecimento por eles de um processo constante de inovação e na valorização do conhecimento de seus especialistas.

A BIMMS não só conta com pessoal especializado nas diversas áreas da indústria de Arquitetura, Indústria, Construção e Operação (AECO), mas também na área de tecnologia da informação e programação. Com ampla experiência no apoio ao Projeto, Construção e já muito desenvolvimento na área de Operação e Manutenção e com o sempre presente objetivo de inovação a empresa tem investido em áreas específicas de desenvolvimento inovador, tais como realidade aumentada, programação de plug-ins para softwares existentes de áreas específicas que atendam às necessidades dos usuários, apoio a teses de mestrado e doutorado, bem como participação em treinamentos BIM a nível nacional e internacional e também com participação em grupos de trabalho nacionais e europeus de padronização BIM.

A aposta na inovação representa uma forte parcela dos custos operacionais da empresa, traduzindo esta confiança em um valor agregado nos serviços oferecidos, que são diferentes em relação à oferta normal do mercado.

\subsection{Fluxo de informação BIM}

No desenvolvimento de um modelo de arquitetura BIM, uma de suas principais bases é a criação e gestão da informação. Nesta acepção, em alguns projetos estudados esse fluxo baseia-se na carência de extrair informações de um modelo BIM, de acordo com as condições legais para o licenciamento de um projeto arquitetônico, consistindo no preenchimento automatizado da tabela sinóptica.

Para o transporte de dados se perscruta a utilização de aplicativos BIM. As trocas de dados entre dois aplicativos BIM são efetuadas basicamente de quatro diferentes maneiras: ligação direta; formato de arquivo proprietário; formato de troca de dados de domínio público e troca de dados baseados em eXtensible Markup Language (XML). (Eastman et 
al., 2008).

Em um possível fluxo de trabalho entre o Revit - Dynamo - Excel. Este é baseado em regras previamente definidas, a fim de facilitar a interligação entre plataformas previamente programadas, que pode ser descrita no protocolo BIM. Assim. é utilizado o Dynamo, que, como ferramenta de programação visual, permite a coleta de dados para o Excel onde são calculados, compilados e tornados acessíveis a todos os participantes, sem a necessidade de utilizar o modelo nativo. Entretanto, como parte destas informações é gerada no Revit de forma isolada, o restante é calculado no Excel, com a necessidade de retornar estas informações ao Revit, novamente utilizando o Dynamo.

Desta maneira, é produzido um fluxo de trabalho automático e explícito, baseado em um modelo BIM, atenuando os possíveis riscos relativos à qualidade da informação gerida e agilizando todo o processo.

\subsection{Caracterização topográfica da infraestrutura de transportes a partir de veículos aéreos não tripulados (Vant)}

A ascensão da tecnologia proporciona um novo e crescente uso de diversos recursos para melhoria de métodos anteriormente utilizados. Atualmente os veículos aéreos não tripulados (VANTS) ganham espaço para inovação da modelagem de e gestão de infraestruturas de transportes.

No cenário atual a utilização de VANTS é torna-se comum inúmeras aplicações, como exemplo na área da construção civil e inspeções em algumas áreas da arquitetura. Além de diversas ajudas humanitárias (como transporte de alimentos e remédios para áreas de difícil acesso).

Avanços recentes na tecnologia computacional, desenvolvimento de software, materiais mais leves, sistemas globais de navegação, avançados links de dados, sofisticados sensores e a miniaturização são os motivos do aumento de desenvolvimentos de VANTs (Jorge et al., 2011, p.399).

Segundo Zaparolli (2019), os drones, pequenas aeronaves que foram criadas em meio militar e hoje se popularizaram, podem medir o perímetro da construção, transmitir coordenadas exatas, gerar imagens aéreas e monitorar os canteiros. Com o tempo a utilização de drones se tornou a forma mais viável de se obter imagens em tempo real das construções, além de chamar mais atenção dos clientes no ramo imobiliário.

Figura 1 - Utilização do sensor Leap Motion na manipulação de objetos BIM no ambiente.

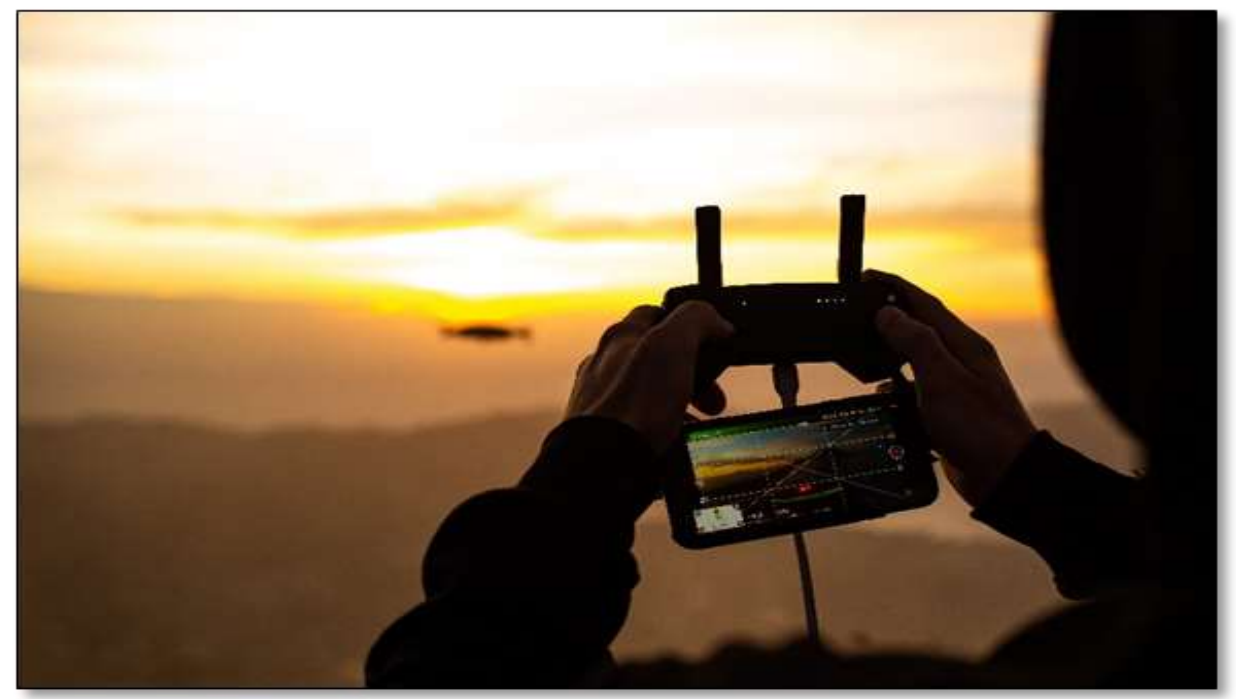

Fonte: https://hompimpasinema.com/jasa-pembuatan-sound-effect-terbaik-di-indonesia/ 
Aplicação de VANT, Fotogrametria e BIM para Infraestrutura urbana e essas novas tecnologias aliadas Modelagem da Informação para Construção de forma prática aplica ao levantamento de dados e a aplicação desses em estudos de concepção de projetos de infraestrutura urbana.

Além disso, é escopo do módulo proporcionar a atualização do aluno para as novas tecnologias da construção 4.0 utilizadas no Brasil no mundo para obras de infraestrutura urbana como a demonstração e prática de softwares BIM, Realidade Virtual, Realidade Aumentada, Scanner Laser, e o uso de informações geográficas precisas baseado no banco de dados.

Figura 2 - Indústria 4.0 de desenvolvimento.

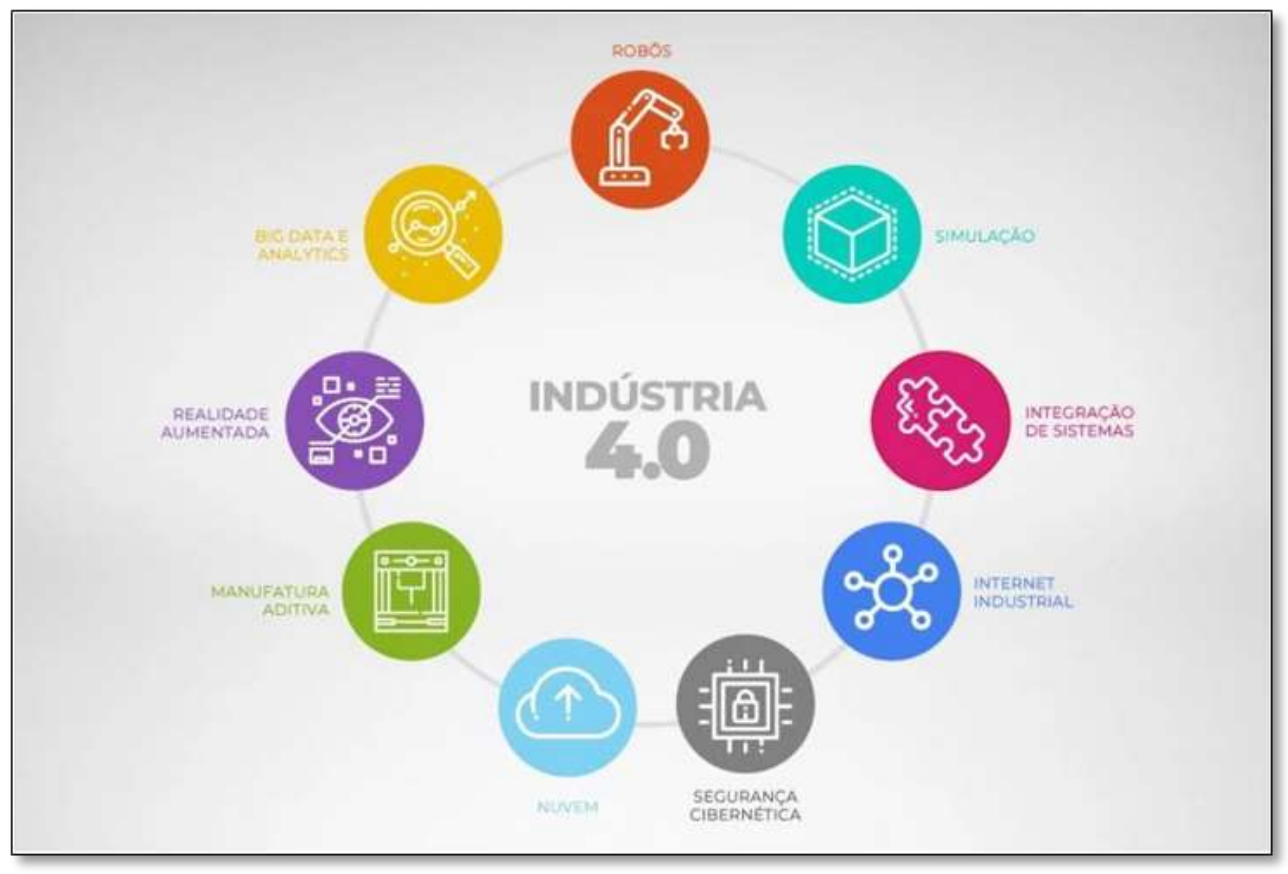

Fonte: https://www.altus.com.br/post/212/conheca-os-nove-pilares-da-industria-4-0-e-sua-relevancia-para-a-atividade-industrial

A aplicação de VANT, Fotogrametria em BIM para Infraestrutura Urbana trás da compreensão dos conceitos práticos da tecnologia BIM e de infraestrutura possibilitando a modelagem preliminar para estudos de viabilidade de um loteamento urbano por meio da integração de softwares e equipamentos de coleta de dados.

\subsection{Uso das ferramentas BIM 4D para preparação, planejamento e acompanhamento de obras}

O desenvolvimento deste artigo envolve a demonstração das vantagens da implementação de metodologias BIM no setor de construção. Quando se trata dos recursos do 4D BIM liga não apenas os aspectos espaciais, mas também os aspectos temporais do projeto, melhorar a confiabilidade dos horários e minimizar os problemas de comunicação, permitindo evitar atrasos no cumprimento de prazos e evitar possíveis ultrapassagens orçamentárias.

O uso do BIM no planejamento 4D, possibilita analisar em detalhes todo o sequenciamento e estudos previstos para a execução do projeto. A plataforma BIM permite modelar não apenas o edifício ou a instalação a ser construída, mas também o processo de construção do edifício ou da própria instalação. É possível modelar as diversas fases de um canteiro de obras, guindastes, elevadores, bandejas de segurança e usar esses modelos para estudar em detalhes todo o processo de construção, definindo a sequência de atividades com um nível de informação sem precedentes. O BIM permite que alguns dos seus softwares permitam a criação de apresentações animadas para demonstradas e exemplificar as atividades previstas no canteiro de obra. 


\subsubsection{Analise das vantagens da compatibilização do BIM 4D}

Construtibilidade: Pode ser definida como a boa utilização do conhecimento e perícia da construção para alcançar o sucesso num desenvolvimento.

Utilização de modelos 4D na construção: A utilização de modelos 4D, que são os modelos 3D gerados pelos desenhadores em diferentes formatos, será incorporada no processo de planeamento e execução, associando os elementos de construção à estrutura de desenho analítico EAP, preparando o modelo para receber mais informação para o planeamento e execução. Entre as várias utilizações dos modelos visuais 4D, podemos destacar o estudo de viabilidade: Possibilidade de realizar uma análise de como a empresa será, processar Processo construtivo, qual é a previsão de entrega e a estimativa de custos.

Planeamento: Definição do plano de ataque da obra, através da simulação de vários cenários: como será a logística do local, especialmente na definição da movimentação e armazenamento de materiais, equipas, equipamentos como gruas, proporcionando uma sequência optimizada de tarefas, e muitos destes elementos são temporários e não farão parte do trabalho em si, como as gruas e outros itens do armazém.

Construção: Para os gestores, será possível conhecer o progresso do projeto, com o estado que indica se está atrasado ou adiantado, além de verificar como será o projeto na linha do tempo numa data prevista. Definir o planeamento da execução semana a semana e a atribuição das equipas.

Monitorização: Ao alimentar os dados no modelo 4D será possível monitorizar o cronograma de execução, gerando no modelo uma visualização tridimensional de que foi planeado com o que será executado, incluindo a animação do progresso da construção. Integração com o Cronograma

Financeiro: Possibilidade de extrair quantidades precisas e ágeis por fases, gerando informação para relatórios financeiros e orçamentos mais realistas e assertivos.

Conceito - BIM 4D - Otimização da execução e tempo da construção: É possível associar os diversos modelos do projeto ao cronograma da construção a ligar tarefas, tempos e gerar um planeamento visual do progresso da obra, proporcionando ao engenheiro de execução ou ao gestor do projeto o acompanhamento do progresso físico de cada etapa. Tudo no "ecrã" do computador, com uma riqueza de informações em tempo real. Esta etapa permite realizar simulações de disposição física e deslocamento no estaleiro de construção, prever situações críticas e minimizar os riscos no que respeita a equipamento e camiões no transporte de materiais.

Execução mais precisa: Com a utilização das ferramentas BIM É possível representar todo esquema gráfico das instalações da construção. Quando associado ao calendário da atividade de construção, permite transmitir as necessidades de espaço e sequenciamento, recursos de mão-de-obra, materiais com entregas associadas, e locais de equipamento. 
Figura 3 - Comparativo Metodologia BIM X CAD - Curva de esforço MacLeamy.

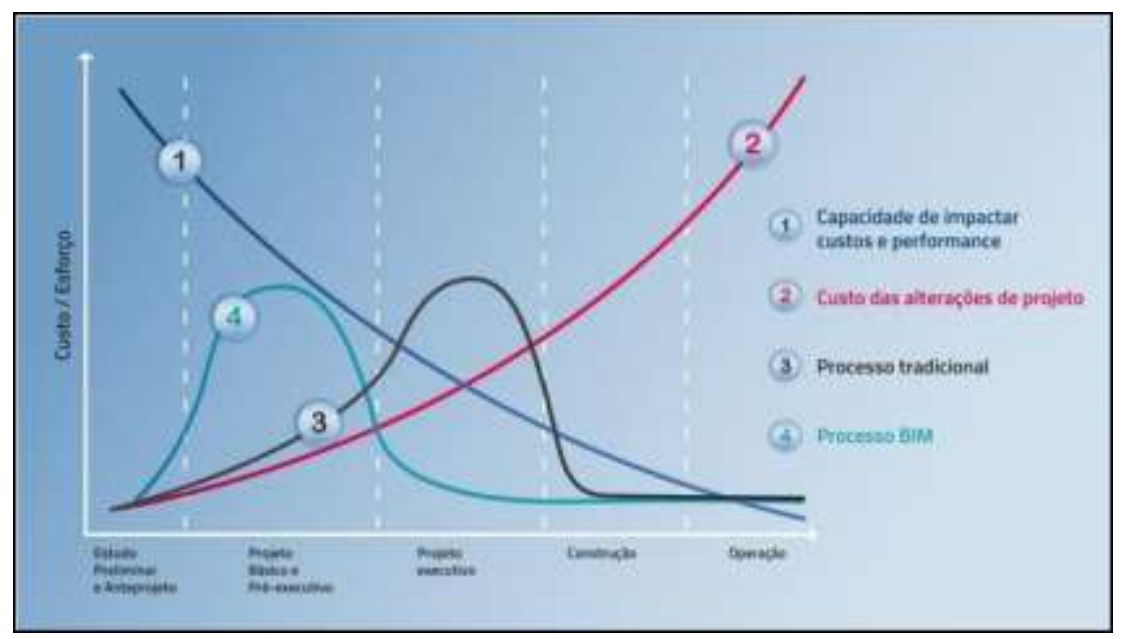

Fonte: Guia AsBEA - Boas práticas em BIM - fascículo II

\subsection{Apresentação de projetos em realidade virtual e aumentada.}

A adoção do BIM (Building Information Modeling) tem sido cada vez maior por parte de empresas de diferentes ramos. Não é difícil entender a motivação dessas corporações: A modelagem 3D permite a visualização e alteração do que está sendo projetado, além do que vários erros já estarão sendo mostrados pelo software. A visualização correta do que está sendo estruturado possibilita a compreensão correta do projeto e isso significa duas coisas: Melhoria no tempo de entrega da obra e durante a fase de construção, uma quantidade menor de problemas.

Vale ressaltar a utilização de novas tecnologias de imersão e interação no setor de Arquitetura e Construção, e os instrumentos para interpretação das possibilidades das mesmas. Uma dessas novas tecnologias BIM é a representação do projeto como realidade virtual (RV) Ainda destaca a importância da utilização dos novos meios de representação e o impacto das apresentações projetuais na hora de defender seus projetos. A RV se apresenta como uma importante ferramenta de marketing e faz paralelo com estratégias de empreendedorismo.

Além de ser útil em lições presenciais, a tecnologia de RV proporciona alternativas fora do comum para um ensino técnico, bem diferente das metodologias tradicionais. 
Figura 13 - Utilização de óculos VR na manipulação de objetos BIM no ambiente.

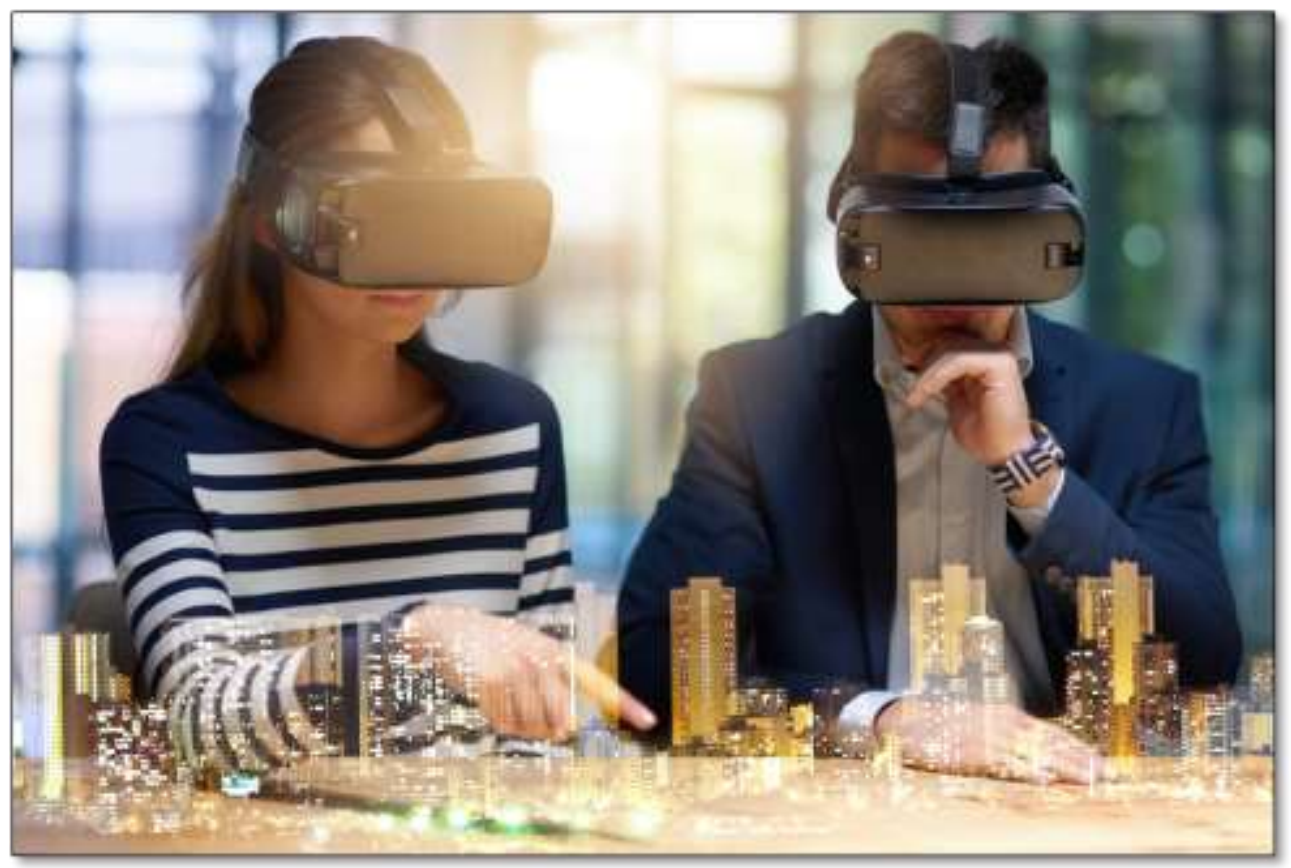

Fonte: https://blogs.autodesk.com/design-studio/2021/10/08/the-smart-city-mobility-infrastructure-opportunity/

\subsection{Realidade virtual na engenharia civil e na indústria da construção}

A possibilidade de "testar" uma construção praticamente num computador, antes de iniciar a execução real no local de construção, é algo realmente valioso para esta indústria.

$\mathrm{Na}$ apresentação do projeto a melhor percepção espacial com o uso do RV pode assumir uma função resolutiva, atuando como um grande intermediador na transmissão de informações entre os colaboradores no processo da construção, demonstrando erros de compatibilização e dificuldades ao longo do processo. Essa inovação tecnológica auxilia projetistas e planejadores nas decisões do início do projeto e na apresentação final. A interface VR pode ser usada em conjunto com vários tipos de software. Sua interface é "simples", e pode ser ajustada para interagir adequadamente com o fundamento específico de cada programa BIM.

Render, é o processo de produzir uma imagem a partir de dados armazenados em um computador/ notebook ou smartphone e agora é uma ferramenta de comunicação visual comumente usada. Uma renderização, é o termo para uma imagem processada, a mesma pode enganar o telespectador por estar confinado ao espaço bidimensional de uma tela uma projeção. Na realidade, a interpretação de uma imagem plana, impressa ou projetada, é sempre recriada pelo de quem quer que esteja vendo e interpretando em 3D. Mesmo se a terceira dimensão do espaço for simulada (imagem plana, usando perspectiva ou 3D), a imersão espacial é inexistente, comprometendo a percepção do espaço.

Por isso é recomendado à RV através de Óculos de realidade Virtual ou realidade aumentada (RA) que é a combinação da captura de imagens com a projeção 3D do projeto, permitindo, através dos elementos gráficos do software a criação da mistura entra o virtual e o mundo real, buscando uma maior interação entre o que está sendo criado e o que pode ser visto.

É necessário um webcam ou outro dispositivo que permita a criação e interpretação do que foi criado virtualmente para que a realidade aumentada (RA) Funcione. Através da câmera, será irradiado a imagem e a animação a serem combinados. Baseado nisso entrará em ação o software para interpretação do que sendo transmitido pela câmera. A figura selecionada será projetada através de uma animação 3D e o aplicativo será responsável pela renderização das ilustrações. A parte mais 
complexa do projeto é verificado através de software dedicado como Revit e seus plug-ins, ArchiCAD, AECOsim, Vectorworks entre outros. Um dos principais objetivos alcançados através do VR e VA é visualizado em paralelo com a execução do projeto em um programa BIM.

\subsection{Dificuldades de implementação}

A necessidade de evolução nos métodos e processos leva a uma busca constante por uma maior eficiência tecnologias mais eficientes, com o objetivo de alcançar a máxima qualidade. A Arquitetura, O setor de Engenharia, Construção e Operação (AECO) deve acompanhar a evolução tecnológica. Por mais heterogêneo que seja o setor, os desvios em termos de prazos e custos não são mais aceitáveis

Muito mais que uma ferramenta de projeto o BIM é uma plataforma de compatibilização de informações e interação tecnológica. Porém, ainda existe muitos entraves para sua implementação, tais como:

- Projetar em 3D durante a fase inicial do projeto:

Já está enraizado na maioria dos profissionais a mentalidade de projetar em $2 \mathrm{~d}$ : A altimetria sempre será após o plano horizontal. Além disso o plano tridimensional sequer muito mais alterações que o plano 2D, logo de acordo com críticos desse processo, isso custará maior tempo.

- Projetos em BIM devem ser usados de maneira simultânea:

A maioria dos projetistas ainda desejam trabalhar de forma linear, que o projeto já esteja totalmente "pronto" para ser iniciado. O que é uma realidade totalmente avessa da metodologia BIM que o processo deve ser colaborativo e simultâneo.

- O processo de coordenação tradicional é muito distinto do processo em BIM:

A verificação dos processos de modelagem e contribuição ainda é um trabalho muito minucioso para profissionais com pouca experiência, ainda é escasso no mercado profissionais com muita experiência em BIM. Na maioria dos processos tradicionais as anotações de projetos são feitas à mão nas folhas de projeto, o que foge da realidade do BIM, já que as anotações são feitas nos modelos 3D.

- Pouca mão de obra qualificada para trabalho em BIM:

Projetistas ainda são poucos qualificados e na grande maioria das vezes essa capacitação é feita de maneira errada, apresentando a metodologia BIM como um software de desenho e não um interoperatividade de projeto

- Dificuldade de compatibilização:

$\mathrm{Na}$ transição de domínio de softwares algumas informações são perdidas, tais como coordenadas globais, escalas, especificações de produtos e matérias. Apesar de conversarem entre si, muitas vezes alguns softwares geram incompatibilidade, gerando perda de tempo nos testes para reconhecimento de erro.

- Fornecedores não possuem biblioteca completa de seus produtos

A falta de visão da indústria para esse seguimento ainda e um entrave para vários projetos, já que o fornecedor não dispõe de uma biblioteca com especificações de componentes, peças e sistemas. Os materiais fornecidos são apenas uma "tendência tecnológica" que servem como um marketing visual.

- Empresas do seguimento da construção civil pouco entendem do BIM.

Como falado anteriormente, a maioria dos projetistas dessas empresas ainda estão "presos" aos modelos tradicionais de modelagem. Para muito o a metodologia BIM serve apenas como uma maquete eletrônica. 


\section{Metodologia}

"A tríade Ciência, Tecnologia e Sociedade (CTS) é uma expressão que define um campo de trabalho acadêmico, o qual tem como objetivo estudar os aspectos sociais da ciência e da tecnologia, abrangendo os aspectos que influenciam na mudança científica e tecnológica, como também as questões sociais e ambientais". (Pereira et al. p.19. 2018.

O presente artigo se descreve como processo de abordagem sistemática e descritivo, respaldando-se em bibliografias, revistas da construção civil, sites referentes ao assunto trazendo como fundo de pesquisa a adaptação do BIM nas industrias e seus impactos na sociedade.

O presente estudo utilizou como metodologia, como base no referencial teórico, revistas da construção civil, plano de implementação do BIM, estudos de casos e a abordagem que o Ministério da Indústria, Comércio Exterior e Serviços trás para auxiliar sobre metas e diretrizes da implementação do BIM no estado do Maranhão e aplicações da tecnologia Bim.

A proposta desse artigo é ter uma temática dinâmica, não havendo apenas uma resposta definitiva e absoluta para o problema. Os estudos se iniciam com uma extensa pesquisa bibliografia sobre os processos de inovação do setor da construção civil, analisando os métodos já adotados atrelando as novas propostas que o BIM traz para o mercado. Em nosso estudo há intenção de trazer informações acerca das funções, processos e participação dos membros envolvidos, assim como uma visão das principais dúvidas que envolvem a implantação da metodologia BIM.

A parte final dos estudos realizados se dará pela interpretação de dificuldades, críticas e interpretação das partes envolvidas para esse processo, de forma que se possa sintetizar os dados analisado. E por fim, apresentar os desafios e resultados que o profissional e empresas da construção civil enfrentam para esta implementação.

\section{Resultados e Discussão}

No contexto da indústria da construção, cada vez mais empresas precisarão de engenheiros com conhecimentos e habilidades para colaborar e se comunicar através das tecnologias BIM. A falta de profissionais qualificados em BIM tem sido citada como um dos principais problemas para a adoção generalizada desta nova metodologia.

$\mathrm{O}$ treinamento de engenheiros mudou de uma posição tradicional, baseada em um currículo que privilegiou a transferência da teoria, para um aprendizado em equipe, focado na solução de problemas com múltiplas soluções, que incluem aplicação prática, assim como especificações e comunicação. Muitos programas de modelagem, apesar de algumas mudanças recentes na sua ementa, ainda são lentos para substituir o projeto tradicional e os processos CAD (2D) por BIM.

Outros cursos de engenharia optam por ligar este assunto apenas a temática de minicursos ou mesmo a cursos de pósgraduação, dando ao BIM a conotação de um assunto complexo ou sofisticado. Vários estudos têm sido realizados para examinar a possibilidade de ensinar esta nova tecnologia em cursos e incentivar seu uso, em paralelo ou mesmo independentemente das tecnologias mais tradicionais, e concluíram que ela pode ser muito útil na transferência de conhecimentos, particularmente na área de compatibilização de resultado, evitando erros.

Neste caso, o modelo BIM não é apenas uma ferramenta agregadora de informações existentes que estão dispersas e em perigo de serem perdidas, cumprindo uma função de maquete digital, mas também pode ser um ponto de acesso às bases de dados existentes para fins de gerenciamento, operação e manutenção. Deve-se notar a qualidade de gestão de dados, e reconhecer suas vantagens, é nítido os privilégios do BIM para demonstrar projetos tridimensionalmente complexos que não podem ser facilmente representadas por modelos tradicionais $2 \mathrm{D}$. $\mathrm{O}$ desenvolvimento da pesquisa apresentado neste artigo nos permitiu concluir que os processos, metodologias e tecnologias BIM podem ser aplicados e proporcionar benefícios para os membros da construção civil. 


\section{Conclusão}

Foi possível notar que adoção do BIM por porte de algumas empresas e profissional como ferramenta de planejamento, gerenciamento de projetos e empreendimentos ainda está engatinhando. Foi observado que a maior dificuldade dos profissionais é a compreensão de precisão de informações trabalhadas nos empreendimentos.

O uso da modelagem tridimensional como forma de apresentação do projeto permite alavancar a produtividade e a qualidade dos resultados adquiridos, desde que exista uma base de trabalho prévia para a preparação da biblioteca paramétrica e dos projetos de edição. Acreditamos que superamos esta fase no que diz respeito a estruturas e arquitetura e que existe agora uma biblioteca paramétrica e rotinas de trabalho que nos permitem avançar na produção de modelos BIM. Entretanto, esta é apenas uma das vantagens do uso deste tipo de tecnologia, e é necessário desenvolver a aplicação do BIM ao projeto. É necessário aproximar as equipes de trabalho do modelo de coordenação, usar a modelagem como ferramenta de projeto e construir um processo de gerenciamento de informação mais eficaz.

O sucesso dos projetos realizados nestas novas formas está intimamente ligado à divulgação e conscientização dos benefícios que estas novas formas trazem. Divulgação e conscientização, entre todos os setores de construção, dos benefícios que podem ser alcançados ao utilizar a metodologia BIM.

Dentre os assuntos abordas e dificuldade apresentadas gostaríamos de continuar com projetos de pesquisa sobre:

(I) A utilização do BIM 4D e 5D enquanto metodologia avançada para planejamento, preparação e monitoramento de obra.

(II) O uso da tecnologia BIM e suas possíveis melhorias em construções no centro histórico de São Luís-MA.

\section{Referências}

Azhar, S., Khalfan, M. \& Maqsood, T. (2012). Building information modeling (BIM): now and beyond. Australasian Journal of Construction Economics and Building.12 $15-28$

Barducco, A. P. S. \& Constância, B. M. (2019). Indústria 4.0: tecnologias emergentes no cenário da construção civil e suas aplicabilidades. Trabalho de Conclusão de Curso apresentado ao Curso de Engenharia Civil da Universidade do Sul de Santa Catarina. Trabalho de Conclusão de Curso apresentado ao Curso de Engenharia Civil da Universidade do Sul de Santa Catarina. 2019.

Boccato, V. R. C. (2006). Metodologia da pesquisa bibliográfica na área odontológica e o artigo científico como forma de comunicação. Rev. Odontol. Univ. Cidade São Paulo, 18(3), 265-274.

Cao, D., et al. (2016), Identifying and contextualising the motivations for BIM implementation in construction projects: An empirical study in China. International Journal of Project Management. Vol. 35, Issue 4, May 2017, P. 658-669.

Cecílio, A. B. G. A. et al. Inovações tecnológicas na construção civil. Revista Científica Multidisciplinar Núcleo do Conhecimento. 12(10), 54-71. Link de acesso: https://www.nucleodoconhecimento.com.br/engenharia-civil/inovacoes-tecnologicas, 10.32749/nucleodoconhecimento.com.br/engenhariacivil/inovacoes-tecnologicas

Chen K., et al. (2015), Bridging BIM and building: From a literature review to an integrated conceptual framework. Int. J. Proj. Manag. International Journal of Project Management. 33(6), 1405-1416

de Andrade, M. L. V. X., \& Ruschel, R. C. (2009). BIM: conceitos, cenário das pesquisas publicadas no Brasil e tendências. Simpósio Brasileiro de Qualidade do Projeto no Ambiente Construído.

Eastman, C. et al. (2008). BIM Handbook: a guide to Building Information Modeling for owners, managers, designers, engineers, and contractors. Hoboken: John Wiley \& Sons, $490 \mathrm{p}$.

Eastman, C., et al (2011), BIM Handbook, in A Guide to Building Information Modeling for Owners, Managers, Designers, Engineers, and Contractors .

Eastman, C.; Teicholz, P.; Sacks, R.; Liston, K. BIM Handbook: a Guide to Building Information Modeling for Owners, Managers, Designers, Engineers, and Contractors. New Jersey:John Wiley \& Sons, 2008.

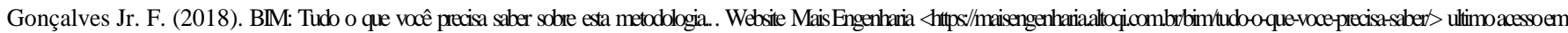
14122021.

Gonçalves Jr. F. (2019). Implantação BIM desafios e por onde começar?. Website <https://maisengenharia.altoqi.com.br/bim/implantacao-bim-desafios-e-por-onde-comecar/> Ultimo acessi 141222021 . 
Research, Society and Development, v. 10, n. 17, e196101724530, 2021 (CC BY 4.0) | ISSN 2525-3409 | DOI: http://dx.doi.org/10.33448/rsd-v10i17.24530

Lino, J. C. Azenha, M. \& Lourenço. P. (2012), Integração da Metodologia BIM na Engenharia de Estruturas, in Encontro Nacional BETÃO ESTRUTURAL BE2012 FEUP

Neil, J. (2014), How has Building Information Modelling changed the MEP Coordination process? University of Salford, Manchester.

Parracho, D. F. R. (2021). Processos digitais para a realização de levantamentos fotogramétricos e termográficos com Veículos Aéreos Não Tripulados (VANT).

Pereira A. S. et al. (2018). Metodologia da pesquisa científica.

Perikamana, A. Ayer, S. K., Beauregard, M. A. \& Alsafouri, S. (2017). "Development of Collaborative Process Mapping Activity to Improve Students' BIM Process Mapping Understanding”. In: LC3 2017: Volume I - Proceedings of the Joint Conference on Computing in Construction (JC3), July 4-7, 2017, Heraklion, Greece, 991-998. https://doi.org/10.24928/JC3-2017/0061.

Quigley, D. E. (2013), Achieving Spatial Coordination Through BIM, MCAA, NECA, and SMACNA.

Victor, A J. (2021) BIM na construção civil é sinônimo de eficiência. <https://grupoajbim.com/category/blog/\#https://grupoajbim.com/category/blog/page/2/> 